\title{
Malignant Maxillary Sinus Neoplasm
}

National Cancer Institute

\section{Source}

National Cancer Institute. Malignant Maxillary Sinus Neoplasm. NCI Thesaurus. Code C3540.

A primary or metastatic malignant neoplasm involving the maxillary sinus. 